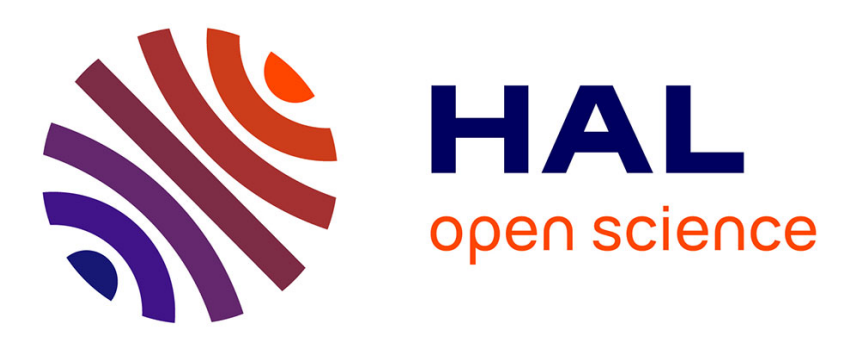

\title{
Towards an Efficient Positioning of Mobile Sinks in Wireless Sensor Networks inside Buildings
}

Leila Ben Saad, Bernard Tourancheau

\section{To cite this version:}

Leila Ben Saad, Bernard Tourancheau. Towards an Efficient Positioning of Mobile Sinks in Wireless Sensor Networks inside Buildings. NTMS - WSN workshop, 2009, Le Caire, Egypt. pp.1 -5. inria00563756

\section{HAL Id: inria-00563756 \\ https://hal.inria.fr/inria-00563756}

Submitted on 8 Feb 2011

HAL is a multi-disciplinary open access archive for the deposit and dissemination of scientific research documents, whether they are published or not. The documents may come from teaching and research institutions in France or abroad, or from public or private research centers.
L'archive ouverte pluridisciplinaire HAL, est destinée au dépôt et à la diffusion de documents scientifiques de niveau recherche, publiés ou non, émanant des établissements d'enseignement et de recherche français ou étrangers, des laboratoires publics ou privés. 


\title{
Towards an Efficient Positioning of Mobile Sinks in Wireless Sensor Networks inside Buildings
}

\author{
Leila Ben Saad, Bernard Tourancheau \\ LIP UMR 5668 of CNRS-ENS-INRIA-Université Lyon1 \\ Lyon, France \\ \{Leila.Ben.Saad, Bernard.Tourancheau\}@ens-lyon.fr
}

\begin{abstract}
Recent years have witnessed an increasing need for wireless sensor networks in a wide range of applications specially for buildings automation. In such networks, many sensor nodes relay the sensed data hop by hop towards the nearest sink. The sensors closest to the sinks drain their energy much faster than distant nodes because they carry heavier traffic which causes prematurely the end of the network lifetime. Relocating the sinks can solve this problem by distributing the high traffic load among the sensors and increase the network lifetime. In this work, we propose a new scalable multi-sink heuristic algorithm (Hop) which regularly moves the sinks towards the distant nodes. We evaluated the performance of our solution by simulations and compared it with others schemes. The results show that it extends significantly the network lifetime and balances notably the energy consumption among the nodes. Such results can provide useful guidelines for real sensor network deployment.
\end{abstract}

Index Terms-Wireless sensor networks, Sinks positioning, Mobile sinks, Network lifetime.

\section{INTRODUCTION}

Wireless sensor networks (WSNs) deployment inside buildings is a very challenging problem. In fact, such networks are formed by a large number of tiny sensors with limited and nonrenewable energy supply. The sensors near the sink are more likely to use up their energy much faster than distant nodes because they carry heavier workloads due to forwarding the data of nodes farther away as well as their own data. Therefore, they become hotspots. The hotspot problem prevents farther nodes to relay their data to the sinks. Consequently, the network lifetime ends prematurely.

In order to solve this problem, many researchers propose to place more sensor nodes around the sink[1][2]. However, these solutions are not always feasible in practice and result in unbalanced sensing coverage over different regions of the network. Another proposed solution is to make multiple fixed sinks cooperate with each other to lighten the load and distribute it among the nodes[3][4]. But, it has been proved in [5] that using a mobile sink is more efficient than a static one and thus helps to increase the network lifetime.

Most of published works suggest to move a single sink to improve the network lifetime[6][7][8][9][10]. But, very few studies focused on the mobility of multiple sinks. Some proposed algorithms find the locations of mobile sinks by solving a mathematical model[11][12]. In [11], the algorithm

This paper has been accepted for publication in the proceedings of WSN Workshop that had been held in conjunction with NTMS 2009. minimizes the average distances between sensors and closest sinks. In [12], the algorithm selects the locations of sinks in the periphery of the network in such way the difference between the maximum and the minimum residual energy of nodes is minimized. To find the optimal placement of mobile sinks, some researches formulate the problem as an Integer Linear Program ILP[13][14][15] or Linear Program LP[16]. However, the drawback of the approaches based on LP and ILP is that they do not scale to thousands of sensors due to their resolution complexity.

Some other works make a moving decision according to the complete knowledge of the energy distribution of the sensors. In [17], the sinks move towards the nodes that have the highest residual energy. But, this strategy requires that the sensors send periodically to the sink additional information about their energy level to allow the sink to found out the nodes which have the highest energy. By doing so, a lot of energy will be wasted.

In this work, we propose a new scalable multi-sink heuristic algorithm (Hop) which regularly moves the sinks towards the distant nodes which have lower load contrary to nodes near the sinks. This approach scales to thousands of nodes and prevents from sending additional information about the energy level of each sensor which is what was done in previous solutions. Simulation results demonstrate that by relocating the mobile sinks according to the proposed algorithm, the energy consumption is balanced among the sensors which lead to significant increase of the network lifetime.

The rest of this paper is organized as follows. In Section 2, we describe the network model including the major assumptions. Section 3 presents our proposed multi-sink heuristic algorithm. Section 4 evaluates the performance of the proposed approach and presents the simulation results. Section 5 concludes the paper.

\section{Network Model}

In order to deploy sensors and sinks inside buildings, we made the following assumptions for the network model.

- We assume $N$ static sensors located in a square grid $(L x L)$ with cells of the same size (see figure 1).

- All sensors have a limited initial energy $e_{0}(\mathrm{~J})$ and a fixed transmission range $r(\mathrm{~m})$ equal to the distance between two nodes (i.e, cell size). 


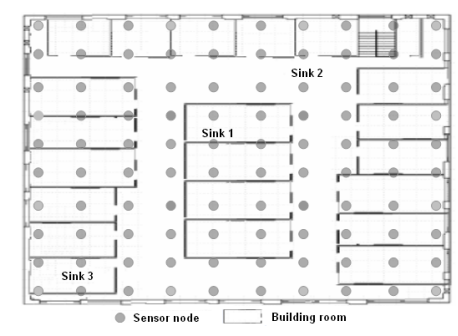

Fig. 1. 10x10 grid of cells with sensors in office building $(L=10)$

- A time-driven application is considered where each sensor regularly generates the same amount of data $g_{r}(\mathrm{bit} / \mathrm{s})$.

- $M$ sinks keep moving in the grid from one node to another one until the network lifetime end.

- The network lifetime is defined as the time until the first sensor dies (i.e, it uses up its residual energy).

- The sinks should stay at a certain location for at least a duration of time $T$ (sojourn time). At the end of this duration, they can change their locations.

- The traveling time of sinks between sensor nodes is considered negligible for analytical simplicity.

- The sensor nodes which are not co-located with any sinks inside the grid, relay their generated data via multiple hops to reach the nearest sink using the shortest path routing protocol.

- In our routing protocol, we consider only the two paths along the perimeter of the rectangle, i.e., paths 1 and 2 in figure 2. These two routes are considered equivalent.

- An ideal MAC layer with no collisions and retransmissions is assumed.

- Only the energy consumption for communication is considered. Let $e_{T}$ (J/bit) be the energy consumption coefficient for transmitting one bit and $e_{R}$ (J/bit) be the energy consumption coefficient for receiving one bit.

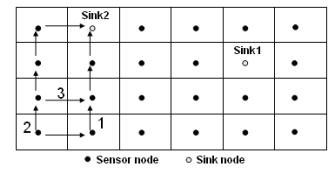

Fig. 2. Path selection

\section{Multi-Sink Heuristic Algorithm (Hop)}

The purpose of the multi-sink heuristic algorithm is to find the best way to move the sinks in order to improve the lifetime of large scale sensor networks. Our approach is based on number of hops and consists in relocating periodically the sinks towards the distant nodes. The difference between our strategy and what was already proposed is that there is no need for the sensors to drain their energy in sending additional information about their energy level. Each sink knows its own position, others sinks positions and the locations of all the sensors. Therefore, from the number of hops to reach the nearest sink, it is possible to guess which sensors are distant and may have more residual energy.
The algorithm begins with an initialization phase where the sinks are placed at their optimal locations in terms of hop counts. Then, for each sensor, the number of hops to reach the nearest sink is computed. Next, the nodes are sorted with decreasing number of hops in order to determine the distant nodes from the sinks. Afterwards, the first sink will be relocated at the farthest node. The second sink will be relocated at the following distant node but respecting the condition that the number of hops between the two new locations must be upper than minimum number of hops $\min _{h o p}$. The third sink is relocated with the same manner in such way the distance between the three new positions of sinks is upper than $\min _{\text {hop }}$. The remaining sinks are relocated with the same way. All the chosen positions of sinks at each period are saved in a list. In the case that the selected positions of sinks where already chosen in previous periods, the algorithm chooses as the first sink location a node which has not been chosen before and determines the locations of the other sinks. If all sensor nodes where already visited by the sinks, the chosen list is emptied. The same operations are repeated at the beginning of each new period $\mathrm{T}$. The pseudocode of the algorithm is as follows.

\section{Multi-Sink Heuristic Algorithm (Hop)}

1: place the $M$ sinks at optimal starting locations in $L x L$ grid 2: add starting locations of the sinks to chosen $_{\text {list }}$

3: min $_{\text {hop }}= \begin{cases}L-1, & M=2 \\ {[2(L-1) /(M-1)],} & M>=3\end{cases}$

4: while new period $\mathrm{T}$ do

5: for $i=1$ to $N$ do

6: nearest $_{K}=$ nearest sink for sensor node $_{i}$

7: $n_{\text {hop }}=$ number of hops between node $_{i}$ and nearest ${ }_{K}$

8: add $\left(\right.$ node $\left._{i}, n_{\text {hop }}\right)$ to node $_{\text {list }}$

9: end for

10: sort nodes of node $_{\text {list }}$ in decreasing order of $n_{\text {hop }}$

11: $p_{1}=$ select first node of node $e_{\text {list }}$ in the sorted order

12: add $p_{1}$ to select list $_{\text {1 }}$

13: for $i=2$ to $M$ do

14: $p_{i}=$ select next node from node list $_{\text {having minimum }}$ number of hops with nodes in select ${ }_{\text {list }}>=$ min $_{\text {hop }}$

15: add $p_{i}$ to select $_{\text {list }}$

16: end for

17: if nodes from select ${ }_{\text {list }}$ not in chosen $_{\text {list }}$ then

18: add nodes from select list $_{\text {to }}$ to chosen $_{\text {list }}$

19: else

20: empty select $t_{\text {list }}$

21: $p_{1}=$ select not chosen node from node list

22: go to line 12

23: end if

24: if chosen $_{\text {list }}$ contains all nodes then

25: empty chosen $_{\text {list }}$

26: end if

27: for $i=1$ to $M$ do

28: move $\operatorname{sink}_{i}$ to node $p_{i}$ from select list $_{\text {. }}$

29: end for

30: end while 


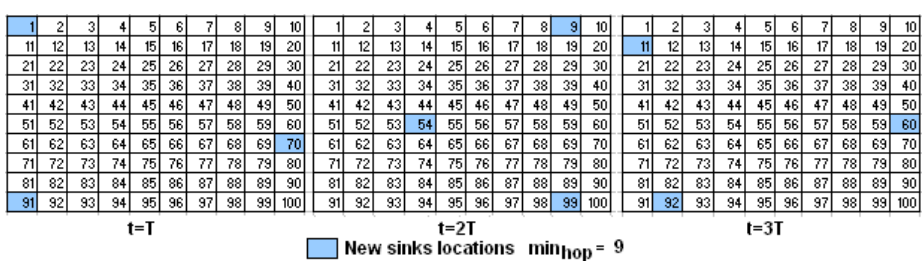

Fig. 3. The sinks locations pattern in $10 \times 10$ grid network $(L=10)$

An example of a network with 100 sensors and 3 mobile sinks is provided. The sinks locations pattern obtained by the execution of our algorithm during the three first periods $\mathrm{T}$ are represented in the figure 3 . The sinks were initially placed at their optimal locations in sensor nodes 15,45 and 86.

\section{Simulation RESUlts}

To analyze the performances of our proposed algorithm, we built a simulator in the Java environment with variable number of sensors and sinks deployed on different grid sizes.

To compute the energy consumption, we used the same model described in [15]. We chose realistic parameter assumptions for the network. The initial energy at each node is equal to energy found in two Alkaline batteries AA of $1.5 \mathrm{~V}$ usually $2600 \mathrm{mAh}$ i.e., $e_{o}=28080 \mathrm{~J}$. The energy consumption coefficient for transmitting and receiving one bit was chosen the same as vendor-specified values for the Chipcon CC2420[18] where $e_{T}=0.22510^{-6} \mathrm{~J} / \mathrm{bit}$ and $e_{R}=0.262510^{-6} \mathrm{~J} / \mathrm{bit}$. We fixed the minimum duration of sojourn time $T$ of the sinks to 30 days because it is economically not easy for technicians to relocate sinks in buildings very often. The sensor transmission range is $r=10 \mathrm{~m}$. The rate at which data packets are generated is $g_{r}=1 \mathrm{bit} / \mathrm{s}$.

To show the efficiency of our proposed scheme, we evaluated its performances by making a comparative study with four other schemes.

Thus, the following schemes were implemented :

1) Static: Static sinks placed in optimal locations[15]

2) Periphery: Sinks moving in the periphery of the network

3) Random: Sinks moving randomly

4) Hop: Sinks moving according to our algorithm

5) Opt: Sinks moving according to ILP solution[15]

In the following sections, the network lifetime, the energy consumption and the residual energy at each sensor are investigated.

\section{A. The network lifetime}

We evaluated the network lifetime of the five schemes on networks of 100 sensors with 2 and 3 mobile sinks.

Figure 4 shows that the Opt scheme performs better than the others schemes because it selects the optimal locations of sinks and their optimal sojourn times. But, the problem with Opt is that it is based on Integer Linear Program which can handle only small scale networks. All the remaining schemes are scalable but the Hop scheme is the best of them because it achieves longer lifetime. The lifetime improvements achieved by Hop in 10x10 grid network with 3 mobile sinks are almost

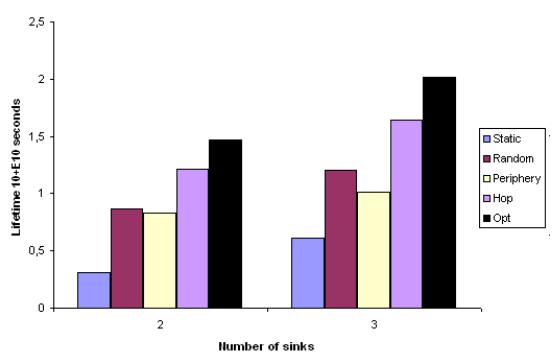

Fig. 4. Network lifetime with 100 sensors (10x10 grid)

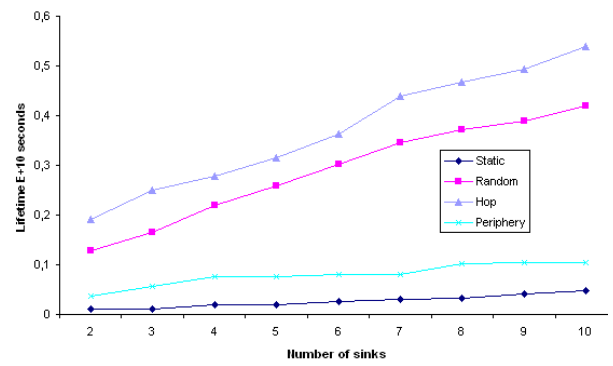

Fig. 5. Network lifetime with increasing number of sinks

$38 \%$ against Random, $64 \%$ against Periphery and almost 170 $\%$ against Static.

We investigated the network lifetime of Static, Random, Periphery and Hop schemes in a large scale network with 2500 sensors on 50x50 grid. We varied the number of sinks from 2 to 10 .

Figure 5 shows that all schemes improve the network lifetime when the number of sinks increases. Because, using more sinks reduces the average path length between the sensors and sinks and enables to achieve less traffic load to the nodes which extends the network lifetime. Nevertheless, the Hop scheme leads to longer network lifetime than all other schemes. In a network with 2500 sensors and 10 sinks, Hop achieves $29 \%$ of lifetime improvement than when 10 sinks are moving randomly, $42 \%$ than when 10 sinks are moving on the periphery, and $1014 \%$ than when 10 sinks are static.

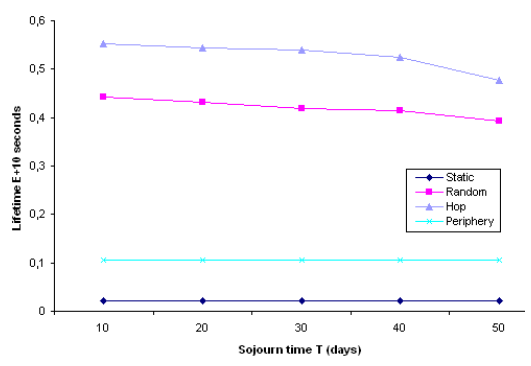

Fig. 6. Network lifetime with different sinks sojourn times

We studied the network lifetime with different periods of sinks sojourn times $T$ (see Figure 6). The number of sinks was fixed to 10 and the number of sensors to 2500. The results indicate that when the sojourn time period of the sinks increases the network lifetime decreases slowly. In fact, the 
longer the sojourn time is, the less the sinks movements are which lead to shorter lifetime.

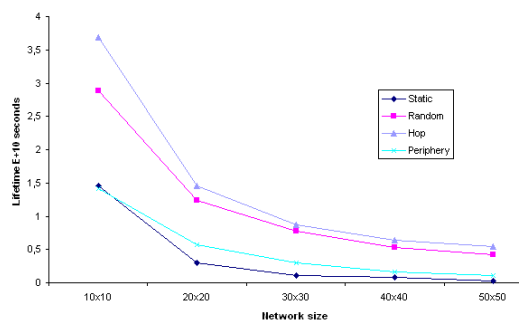

Fig. 7. Network lifetime with increasing network size

We varied the network size from $10 \times 10$ to $50 \times 50$ sensors. The number of sinks was fixed to 10 and the period sojourn time $\mathrm{T}$ to 30 days. We noticed as shown in the Figure 7 that the network lifetime decreases considerably when the network size increases. In fact, the sensors near the sinks must retransmit a higher number of packets from their higher number of neighbors which leads to higher energy consumption.

\section{B. The energy distribution}

We analyzed the impact of the five schemes on the energy consumption at lifetime end in a network with 3 mobile sinks and 100 sensors. The distribution of energy consumption when the first sensor dies is depicted in Figures 8(a), 9(a), 10(a), 11(a) and 12(a). A light color means a higher percentage of energy consumption.

It is remarkable in all the figures that the energy consumption is highly variable and depends on the sinks locations. We notice that the nodes closest the sinks locations have relatively higher energy consumption compared to most of the others because they have to receive and relay all other neighbors data in addition to their own data. This leads them to consume more energy.

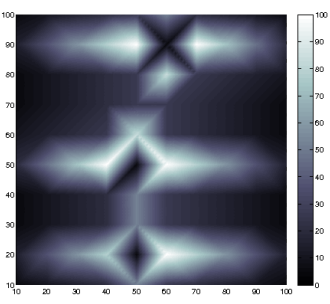

(a) Energy consumption

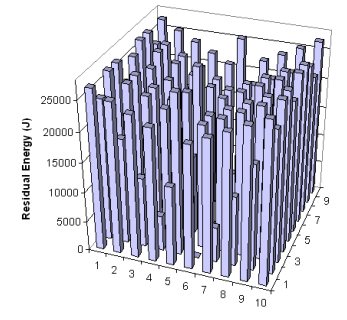

(b) Residual energy
Fig. 8. Static scheme in 10x10 grid network

In figure 8(a), we observe that higher percentage of energy consumption is concentrated around three nodes in the grid which are the locations of the sinks whereas the others sensors have a lower amount of energy consumption (dark color).

When the sinks move on the periphery of the network the highest energy consumption occurs in nodes closest to the boundary of the network while the others nodes specially in the center consume less energy as seen in the Figure 9(a).

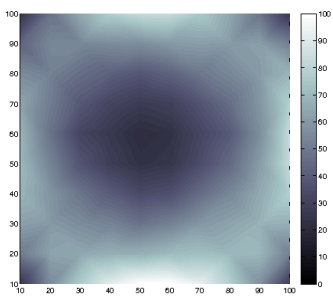

(a) Energy consumption

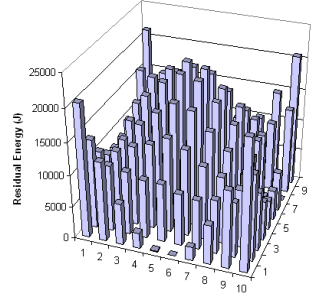

(b) Residual energy
Fig. 9. Periphery scheme in 10x10 grid network

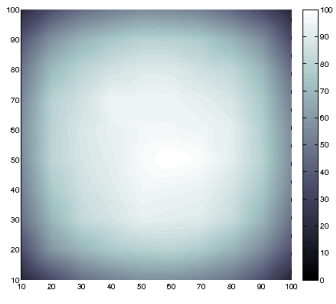

(a) Energy consumption

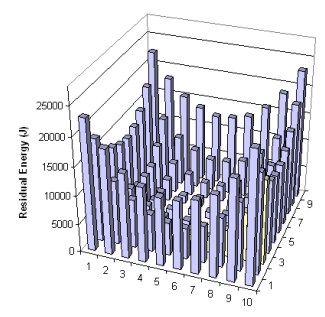

(b) Residual energy
Fig. 10. Random scheme in 10x10 grid network

Figure 10(a) shows that the energy consumption of Random scheme is more balanced among the nodes than Periphery and Static schemes.

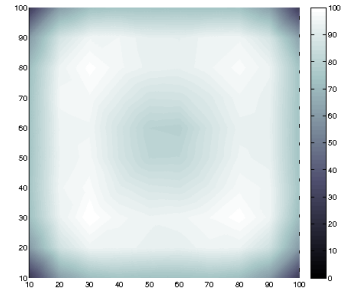

(a) Energy consumption

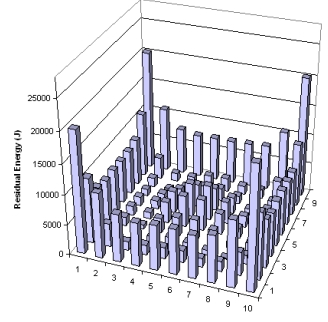

(b) Residual energy
Fig. 11. Hop scheme in 10x10 grid network

Figure 11(a) shows that Hop scheme results in a better balancing of energy consumption than Periphery, Static and Random schemes. In fact, we notice a larger area with light color.

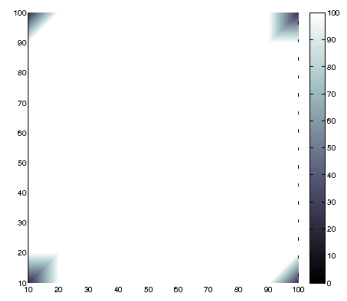

(a) Energy consumption

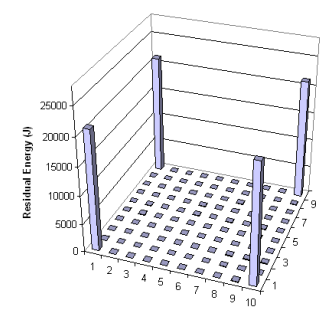

(b) Residual energy
Fig. 12. Opt scheme in 10x10 grid network 
Opt scheme balances almost perfectly the energy consumption among the nodes. In fact, the majority of the nodes deplete their energy at the same time except the four nodes in corners as shown in the Figure 12(a). However, this scheme is restricted only to small scale networks.

The distribution of the residual energy at each sensor node in 10x10 grid with 3 mobile sinks was also studied (see figures 8(b), 9(b), 10(b), 11(b) and 12(b)). The results show that the percentages of the residual energy that remained unused at the network lifetime end are $71 \%, 45 \%, 31 \%, 17 \%$ and $3 \%$ respectively for Static, Periphery, Random, Hop and Opt schemes. Moreover, the numbers of sensors which have more than $50 \%$ of their initial energy at network lifetime end are 80, 36, 20, 4 and 4 respectively for Static, Periphery, Random, Hop and Opt schemes.

The two schemes Opt and Hop result in a better distribution of residual energy compared to Periphery, Random and Static. Nevertheless, Opt can be efficient only for small networks contrary of Hop which can scale to thousands of sensors.

We analyzed the impact of the scalable schemes Static, Periphery, Random and Hop on the energy consumption at lifetime end in a large scale network with 4 mobile sinks and 2500 sensors. The results are similar to what was found in small network with 100 sensors. Hop balances better the energy consumption among the nodes than the other schemes because it leads to a higher number of sensors with very small amount of energy left unused (see the Figure 13).

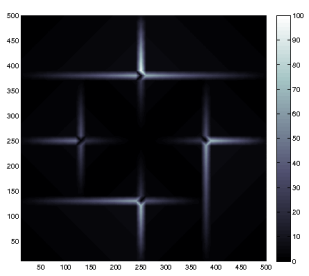

(a) Static

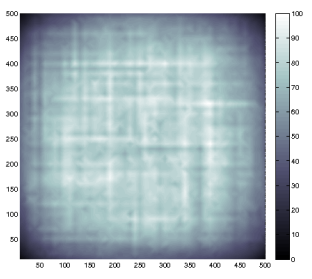

(c) Random

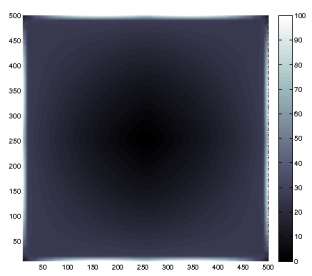

(b) Periphery

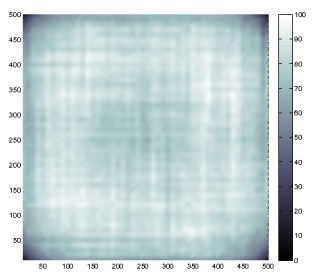

(d) Hop
Fig. 13. Energy consumption in 50x50 grid network

\section{CONClusion}

In this paper, we have proposed an efficient solution to extend the lifetime of large scale WSNs. The proposed heuristic algorithm regularly moves the sinks towards the distant nodes. This approach, based on number of hops, prevents from sending additional information about the energy level of each sensor. We evaluated the performance of our algorithm by simulation in a network with thousands of sensors and compared it with others schemes: Static, Periphery, Random and Opt. The results show that it extends significantly the lifetime of the network and balances notably the energy consumption among the nodes. Our solution is very simple and useful for wireless sensor network deployment inside buildings because it is scalable and achieves $1014 \%$ lifetime improvement when we deploy 10 mobile sinks instead of 10 static ones in a network with 2500 sensors.

\section{REFERENCES}

[1] W. Xiaobing, C. Guihai, and K. D. Sajal, "Avoiding energy holes in wireless sensor networks with nonuniform node distribution," IEEE Transactions on Parallel and Distributed Systems, 2008.

[2] X. Wu, G. Chen, and S. K. Das, "On the energy hole problem of nonuniform node distribution in wireless sensor networks," IEEE International Conference on Mobile Adhoc and Sensor Systems MASS, 2006.

[3] H. Kim, Y. Seok, N. Choi, Y. Choi, and T. Kwon, "Optimal multi-sink positioning and energy-efficient routing in wireless sensor networks," Lecture Notes in Computer Science LNCS, 2005.

[4] E. Oyman and C. Ersoy, "Multiple sink network design problem in large scale wireless sensor networks," IEEE International Conference on Communications, vol. 6, pp. 20-24, 2004.

[5] J. Luo and J.-P. Hubaux, "Joint mobility and routing for lifetime elongation in wireless sensor networks," In Proceedings 24th Annual Joint Conference of the IEEE Computer and Communications Societies INFOCOM, 2005

[6] B. Wang, D. Xie, C. Chen, J. Ma, and S. Cheng, "Employing mobile sink in event-driven wireless sensor networks," IEEE Vehicular Technology Conference VTC, 2008.

[7] Z. M. Wang, S. Basagni, E. Melachrinoudis, and C. Petrioli, "Exploiting sink mobility for maximizing sensor networks lifetime," Proceedings of the 38th Annual Hawaii International Conference on System Sciences HICSS, 2005.

[8] K. Akkaya and M. Younis, "Sink repositioning for enhanced performance in wireless sensor networks," Elsevier Computer Networks Journal, 2005.

[9] S. Basagni, A. Carosi, E. Melachrinoudis, C. Petrioli, and Z. M. Wang, "Controlled sink mobility for prolonging wireless sensor networks lifetime," Wireless Networks, 2007.

[10] Y. Bi, J. Niu, L. Sun, W. Huangfu, and Y. Sun, "Moving schemes for mobile sinks in wireless sensor networks," Performance, Computing, and Communications Conference, 2007.

[11] Z. Vincze, R. Vida, and A. Vidacs, "Deploying multiple sinks in multi-hop wireless sensor networks," IEEE International Conference on Pervasive Services, 2007.

[12] A. Azad and A. Chockalingam, "Mobile base stations placement and energy aware routing in wireless sensor networks," IEEE Wireless Communications and Networking Conference WCNC, 2006.

[13] S. R. Gandham, M. Dawande, R. Prakash, and S. Venkatesan, "Energy efficient schemes for wireless sensor networks with multiple mobile base stations," The IEEE Global Telecommunications Conference GLOBECOM, vol. 1, 2003.

[14] W. Alsalih, S. Akl, and H. Hassanein, "Placement of multiple mobile base stations in wireless sensor networks," IEEE International Symposium on Signal Processing and Information Technology, 2007.

[15] L. Ben Saad and B. Tourancheau, "Multiple mobile sinks positioning in wireless sensor networks for buildings," in The Third International Conference on Sensor Technologies and Applications SensorComm, 2009.

[16] S. Basagni, A. Carosi, C. Petrioli, and C. Phillips, "Moving multiple sinks through wireless sensor networks for lifetime maximization," in 5th IEEE International Conference on Mobile Ad Hoc and Sensor Systems MASS, 2008.

[17] M. Marta and M. Cardei, "Improved sensor network lifetime with multiple mobile sinks," Pervasive and Mobile computing, 2009.

[18] Chipcon. CC2420 2.4 GHz IEEE 802.15.4 / ZigBee-ready RF transceiver, 2004. 